\title{
Casimir effect of two conducting parallel plates in a general weak gravitational field
}

\author{
Borzoo Nazari ${ }^{\mathrm{a}}$ \\ Faculty of Engineering Science, College of Engineering, University of Tehran, P.O. Box 11155-4563, Tehran, Iran
}

Received: 8 August 2015 / Accepted: 13 October 2015 / Published online: 23 October 2015

(C) The Author(s) 2015. This article is published with open access at Springerlink.com

\begin{abstract}
We calculate the finite vacuum energy density of the scalar and electromagnetic fields inside a Casimir apparatus made up of two conducting parallel plates in a general weak gravitational field. The metric of the weak gravitational field has a small deviation from flat spacetime inside the apparatus, and we find it by expanding the metric in terms of small parameters of the weak background. We show that the metric found can be transformed via a gauge transformation to the Fermi metric. We solve the Klein-Gordon equation exactly and find mode frequencies in Fermi spacetime. Using the fact that the electromagnetic field can be represented by two scalar fields in the Fermi spacetime, we find general formulas for the energy density and mode frequencies of the electromagnetic field. Some well-known weak backgrounds are examined and consistency of the results with the literature is shown.
\end{abstract}

\section{Introduction}

The quantum vacuum is a fundamental concept in theoretical physics and its properties have been widely investigated in the literature of quantum gravity and string theory. The theory of quantum fields in curved spacetime, which is believed to be the low energy limit of the ultimate theory of quantum gravity, has predicted famous quantum effects in the presence of gravity. In general, due to the lack of the global symmetries in the spacetime manifold, quantum effect considerations in curved spacetime are mainly limited to the analysis of local quantities such as the vacuum expectation value of the energy-momentum tensor, i.e. $\left\langle\left|T_{\mu \nu}(x)\right|\right\rangle$, in some point $x$. In fact, the most famous results of the semi-classical theory of gravity, like the Hawking radiation and the particle production in the expanding universe, has been achieved from the analysis of $\left\langle\left|T_{\mu \nu}\right|\right\rangle$ in the related curved backgrounds. The most famous vacuum state effect is the Casimir effect. An

\footnotetext{
a e-mail: borzoo.nazari@ut.ac.ir
}

important aspect in the research as regards the Casimir effect in curved spacetime is that the characteristics of the vacuum state are apparently dependent to the geometry of the background spacetime. We are also motivated to see explicitly such a dependency in this paper. Furthermore, computation of the energy, i.e. $\left\langle\left|T_{00}\right|\right\rangle$, has also been done for large number of problems in various spacetimes and in some cases $[1,2]$ has helped us to confirm the validity of the principle of correspondence in the context of the Casimir effect. Finding the total gravitational force on a set of two conducting Casimir plates [1,2] is a typical example. So, due to the importance of the stress-energy tensor, we will consider the $\left\langle\left|T_{\mu \nu}\right|\right\rangle$ for the plates in a general weak background.

The Casimir effect arises when there is a boundary in our problem and it predicts a force between two uncharged conducting metals in the presence of a quantum field. The effect has been measured to great accuracy $[3,4]$. We use the zeropoint energy approach here, although it is possible to find the Casimir force and energy without any reference to the zero-point energy [5]. We may also have the Casimir effect without having a boundary at all. In fact, some non-trivial topologies in curved spacetime do the same job as a boundary does $[6,7]$. The Casimir energy in curved spacetime has also been analyzed by many authors ([1,2,6,8-25] and references therein). Recently, a Casimir apparatus consisting of two ideal conducting parallel plates in the weak field limit of the Kerr and the Horava-Lifshitz spacetimes has been studied in [8-11]. A purpose of this paper is to generalize the above analysis for a scalar field finding an exact solution of the Klein-Gordon equation in a general weak gravitational field. Also we extend the method for the case that an electromagnetic field is present inside the plates.

The organization of the paper is as follows. In Sect. 2 using the fact that the apparatus is composed of tiny pales, we will find the small deviations of the metric from flat spacetime inside the apparatus. In fact, we will expand the metric up to first order in terms of the parameters of the general weak gravitational field. In Sect. 3 the Klein-Gordon equation will be 


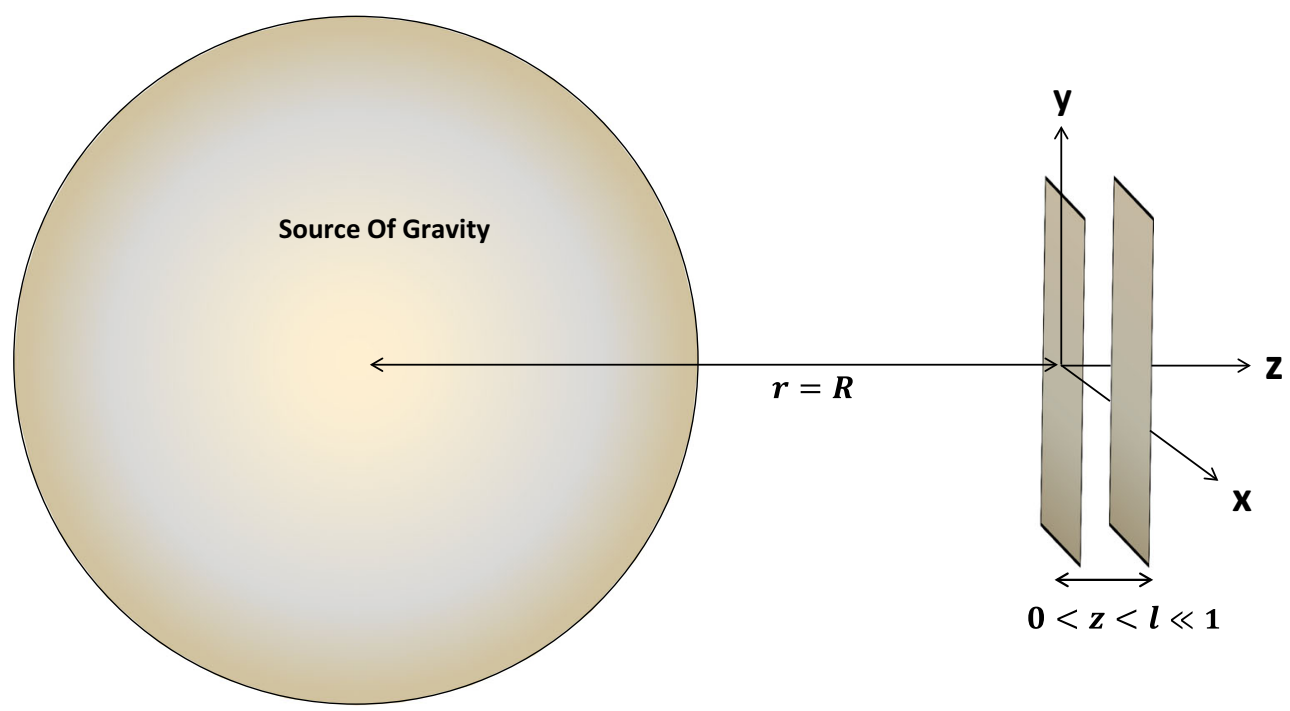

Fig. 1 The Casimir apparatus far from the center of the source of a weak gravitational field. The $z$ axis coincides with the $r$ axis in the equatorial plane and the plates are separated by a small coordinate distance $l<<1$. The boundaries are at $z=0, z=l$

solved exactly inside the apparatus using the metric obtained in the previous section. In Sect. 4 mode frequencies inside the apparatus will be obtained under the influence of both Dirichlet and Neumann boundary conditions for the scalar field. A generalization to the case where the electromagnetic field is present will be done using an interesting property of the Fermi spacetime in Sect. 5. Computation of the energymomentum tensor for the scalar field for both Neumann and Dirichlet boundary conditions is performed in Sect. 6. Also the electromagnetic energy density is obtained in this section. Well-known weak gravitational fields are examined in Sect. 7. The electromagnetic energy density for the far field limit of the Kerr spacetime and the Horava-Lifschitz theory of gravity are of special interest. The final section is devoted to the conclusion.

\section{Transformation of the metric of a weak gravitational field into the Fermi metric}

In the $1+3$ formalism of general relativity, the stationary spacetime metric is defined by ${ }^{1}$

$\mathrm{d} s^{2}=g_{00}\left(\mathrm{~d} x^{0}-A_{i} \mathrm{~d} x^{i}\right)^{2}-\mathrm{d} l^{2}$,

where $A_{i}=-\frac{g_{0 i}}{g_{00}}$ is the so-called gravitomagnetic potential and

$$
\begin{aligned}
\mathrm{d} l^{2} & =\gamma_{i j} \mathrm{~d} x^{i} \mathrm{~d} x^{j} \\
& =\left(-g_{i j}+\frac{g_{0 i} g_{0 j}}{g_{00}}\right) \mathrm{d} x^{i} \mathrm{~d} x^{j}, \quad i, j=1,2,3 .
\end{aligned}
$$

\footnotetext{
${ }^{1}$ See [26] for more discussions on the gravitomagnetic effects in the stationary spacetimes. Also see $[27,28]$.
}

In the weak field slowly rotating limit $(\Phi<<1, v<<c)$, the metric (1) is equivalent to

$\mathrm{d} s^{2} \approx\left(1+\frac{2 \Phi}{c^{2}}-\frac{2 \mathbf{A} \cdot \mathbf{v}}{c^{2}}\right) c^{2} \mathrm{~d} t^{2}-\left(1-\frac{2 \Phi}{c^{2}}\right) \delta_{i j} \mathrm{~d} x^{i} \mathrm{~d} x^{j}$

The explicit form of a general weak gravitational field line element is as follows (see (19.13) in [29]):

$$
\begin{aligned}
\mathrm{d} s^{2}= & {\left[1-\frac{2 M}{r}+\frac{2 M^{2}}{r^{2}}+O\left(\frac{1}{r^{3}}\right)\right] \mathrm{d} t^{2} } \\
& -\left[4 \epsilon_{i j k} S^{j} \frac{x^{k}}{r^{3}}+O\left(\frac{1}{r^{3}}\right)\right] \mathrm{d} x^{i} \mathrm{~d} t \\
& -\left\{\left(1+\frac{2 M}{r}+\frac{3 M^{2}}{2 r^{2}}\right) \delta_{i j}\right. \\
& \left.+ \text { gravitational radiation terms that die out as } O\left(\frac{1}{r}\right)\right\} \mathrm{d} x^{i} \mathrm{~d} x^{j} .
\end{aligned}
$$

A comparison between (3) and (4) shows that $\Phi=-\frac{G M}{r}$ is the newtonian potential, $A^{i}=\epsilon_{i j k} S^{j} \frac{x^{k}}{r^{3}}$ is the gravitomagnetic potential, and $\mathrm{v}^{i}=\frac{\mathrm{d} x^{i}}{\mathrm{~d} t}$. We need to have an isotropic coordinate representation of the metric in the next sections, and Eqs. (1), (2), and (3) show us the way we can construct an isotropic coordinate representation of any weak gravitational field.

Figure 1 shows the apparatus in a weak gravitational field. Two ideal conducting plates are at a small coordinate distance $l$ from each other. Inside the plates, the metric has a small variation relative to the flat spacetime metric. To find this variation, we adapt a rectangular coordinate system having the origin at one of the plates (the one which is closer to the source and having coordinate distance $R$ from it) and expand 
the metric (4) inside the apparatus in the neighborhood of the point $r=R$. The overall size of the apparatus is so small that we can assume $r=R+z$ :

$$
\begin{aligned}
g_{\mu \nu}(r, \theta) & =\eta_{\mu \nu}+h_{\mu \nu}(R+z, \theta) \\
& =\eta_{\mu \nu}+h_{\mu \nu}(R, \theta)+\left.\frac{\mathrm{d} h_{\mu \nu}(r, \theta)}{\mathrm{d} r}\right|_{z=0} z+O\left(\epsilon z^{2}\right),
\end{aligned}
$$

in which $R>>z, \quad h_{\mu \nu}<<1$. For the case of a static spacetime, the components of the metric (5) can be written in the following form:

$g_{\mu \nu}=1+2 \gamma+2 \lambda z+O\left(\gamma z^{2}\right)$

where $\gamma<1, \lambda<1$ are constants and use is made of $\Phi=$ $\gamma+\lambda z+O\left(\gamma^{2}\right), \gamma=\frac{-G m}{R}, \lambda=\frac{G m}{R^{2}}$. Concerning the form of $A^{i}$, the above expansion satisfies $\left.\frac{\mathrm{d} h_{\mu \nu}(R, \theta)}{\mathrm{d} r}\right|_{z=0}<1$ provided that $\theta=\pi / 2$, i.e. in the equatorial plane. So (5) and (6) are also valid for the case of the far field limit of the Kerr spacetime and we will get back to it in the examples in Sect. 6.

Motivated by the above discussion, in this paper we analyze the general case of the spacetime of the form

$$
\begin{aligned}
\mathrm{d} s^{2}= & \left(1+2 \gamma_{0}+2 \lambda_{0} z\right) \mathrm{d} t^{2}-\left(1+2 \gamma_{1}+2 \lambda_{1} z\right)\left[\mathrm{d} x^{2}\right. \\
& \left.+\mathrm{d} y^{2}+\mathrm{d} z^{2}\right],
\end{aligned}
$$

in which $\gamma_{0}, \lambda_{0}, \gamma_{1}, \lambda_{1}<1$.

To solve the Klein-Gordon equation, it is better to recast the metric (7) in the form of the well-known Fermi metric. We use the linearized weak field regime of general relativity and change the variables with the aid of the following gauge transformation:

$$
\begin{aligned}
g_{\mu \nu} & =\eta_{\mu \nu}+h_{\mu \nu}, \quad\left|h_{\mu \nu}\right|<<1, \\
h_{\mu \nu}^{\prime} & =h_{\mu \nu}-\zeta_{\mu, \nu}-\zeta_{\nu, \mu}, \\
x^{\prime \mu} & =x^{\mu}+\zeta^{\mu}, \\
\zeta_{t} & =\left(\gamma_{0}+\lambda_{0}\left(z-z^{\prime}\right)\right) t, \zeta^{t}=\left(\gamma_{0}+\lambda_{0}\left(z-z^{\prime}\right)\right) t, \\
\zeta_{x} & =-\left(\gamma_{1}+\lambda_{1} z\right) x, \zeta^{x}=\left(\gamma_{1}+\lambda_{1} z\right) x, \\
\zeta_{y} & =-\left(\gamma_{1}+\lambda_{1} z\right) y, \zeta^{y}=\left(\gamma_{1}+\lambda_{1} z\right) y, \\
\zeta_{z} & =-\gamma_{1} z-\lambda_{1} z^{2}, \zeta^{z}=\gamma_{1} z+\frac{1}{2} \lambda_{1} z^{2},
\end{aligned}
$$

in which we have assumed $h_{i j}^{\prime}=0$ to force the spatial sector of the metric (7) to be flat. More explicitly we have

$$
\begin{aligned}
& t^{\prime}=t+\gamma_{0} t, \\
& x^{\prime}=x+\left(\gamma_{1}+\lambda_{1} z\right) x, \\
& y^{\prime}=y+\left(\gamma_{1}+\lambda_{1} z\right) y, \\
& z^{\prime}=z+\gamma_{1} z+\frac{1}{2} \lambda_{1} z^{2} ;
\end{aligned}
$$

the metric then takes the following form up to first order in the parameters $\gamma_{0}, \lambda_{0}, \gamma_{1}, \lambda_{1}$ :

$$
\mathrm{d} s^{2}=\left(1+2 \lambda 0 z^{\prime}\right) \mathrm{d} t^{2}-\mathrm{d} x^{\prime 2}-\mathrm{d} y^{\prime 2}-\mathrm{d} z^{\prime 2} .
$$

The gauge transformation, however, changes our primary problem as follows. In fact according to the last equation in (8) the boundaries must be transformed from $z=0$ and $z=l$ in the spacetime (7) to $z^{\prime}=0$ and $z^{\prime}=l+\gamma_{1} l+\frac{1}{2} \lambda_{1} l^{2}$ in the spacetime (10). Another change that the gauge transformation brings into the problem is the rescaling of time in (9) by the factor $1+\gamma_{0}$. This corresponds, in turn, to dividing the mode frequencies $\omega$ by $1+\gamma_{0}$ because of the presence of the factor $\mathrm{e}^{-i \omega t}$ in our solution of the Klein-Gordon equation in the next section. So time must be re-inverted after solving the Klein-Gordon equation when we obtain the mode frequencies. The net effect of the rescaling of time is that the final mode frequencies must be multiplied by the factor $1+\gamma_{0}$. We drop the dashes _ on $x^{\prime}, y^{\prime}, z^{\prime}$ from now on using the new boundary conditions instead.

\section{Exact solution to the massless Klein-Gordon equation in the Fermi metric}

The massless Klein-Gordon equation is

$\partial_{\mu}\left[\sqrt{-\mathfrak{g}} g^{\mu \nu} \partial_{\nu} \Phi\left(x^{c}\right)\right]=0, \quad \mathfrak{g} \equiv \operatorname{det} g^{\mu \nu}$.

Since the spacetime is spatially flat we assume the following form for the solution:

$\Phi(x)=C \mathrm{e}^{-i \omega t} \mathrm{e}^{i k_{x} x} \mathrm{e}^{i k_{y} y} Z(z)$,

where $C$ is normalization constant determined through the commutation relations

$\left(\Phi_{i}(x), \Phi_{j}(x)\right)=\delta_{i j} \delta\left(\mathbf{k}_{i}-\mathbf{k}_{j}\right)$.

The scalar product is defined as

$\left(\Phi_{1}, \Phi_{2}\right)=-i \int_{\Sigma} \Phi_{1}(x) \overleftrightarrow{\partial}_{\mu} \Phi_{2}^{*}(x)\left[-\mathfrak{g}_{\Sigma}(x)\right]^{\frac{1}{2}} n^{\mu} \mathrm{d} \Sigma$

in which $n_{\mu}=\partial_{\mu} z$ and $\mathrm{d} \Sigma$ spans the space between the plates. Under the above assumptions Eq. (11) reads

$$
(1+2 \lambda z) Z^{\prime \prime}(z)+\lambda Z^{\prime}(z)+\left(\omega^{2}-(1+2 \lambda z) k_{\perp}^{2}\right) Z(z)=0,
$$

where' denotes derivation with respect to $\mathrm{z}$ and $k_{\perp}^{2}=k_{x}^{2}+k_{y}^{2}$. Another variable change $V(z)=\frac{Z(z)}{\sqrt{1+2 \lambda z}}$ yields

$$
(1+2 \lambda z) V^{\prime \prime}(z)+3 \lambda V^{\prime}(z)+\left(\omega^{2}-(1+2 \lambda z) k_{\perp}^{2}\right) V(z)=0 .
$$

The appearance of the factor 3 in front of the second term introduces a significant simplification when we change the variable to $T(z)=\exp \left(k_{\perp} z\right) V(z)$. It recasts (16) into

$$
\begin{aligned}
(1+2 \lambda z) T^{\prime \prime}(z) & +\left(3 \lambda-2 k_{\perp}(1+2 \lambda z)\right) T^{\prime}(z) \\
& +\left(\omega^{2}-3 \lambda k_{\perp}\right) T(z)=0 .
\end{aligned}
$$


A simple reparametrization of this last equation via $u=$ $\frac{k_{\perp}}{\lambda}(1+2 \lambda z)$ leads to

$u T^{\prime \prime}(u)+\left(\frac{3}{2}-u\right) T^{\prime}(u)+\left(\frac{\omega^{2}}{4 k_{\perp} \lambda}-\frac{3}{4}\right) T(u)=0$.

This is the well-known Kummer differential equation

$u T^{\prime \prime}(u)+(B-u) T^{\prime}(u)-A T(u)=0$,

in which $A=\frac{3}{4}-\frac{\omega^{2}}{4 k_{\perp} \lambda}, B=\frac{3}{2}$. Kummer's differential equation is not suitable for next considerations and we transform it via $T(u)=u^{-\frac{B}{2}} e^{\frac{u}{2}} W(u)$ to another well-known form, called Wittaker's differential equation:

$W^{\prime \prime}(u)+\left(-\frac{1}{4}+\frac{B-2 A}{2 u}+\frac{\frac{1}{4}-\mu^{2}}{u^{2}}\right) W(u)=0$

in which in our case $\mu=\frac{B-1}{2}=\frac{1}{4}, \kappa \equiv \frac{B-2 A}{2}=\frac{\omega^{2}}{4 k_{\perp} \lambda}$. Equation (20) has two independent sets of solutions $M_{\kappa, \mu}$, $W_{\kappa, \mu}$ and their asymptotic behavior is as follows [30]:

$M_{\kappa, \mu}(u)= \begin{cases}\Gamma(1+2 \mu) \mathrm{e}^{\frac{u}{2}} u^{-\kappa} & u \rightarrow \infty \\ u^{\frac{3}{4}} & u \rightarrow 0,\end{cases}$

$W_{\kappa, \mu}(u)= \begin{cases}\mathrm{e}^{-\frac{u}{2}} u^{\kappa} & u \rightarrow \infty \\ \frac{\Gamma(2 \mu)}{\Gamma\left(\frac{1}{2}+\mu-\kappa\right)} u^{\frac{1}{2}-\mu} & u \rightarrow 0 .\end{cases}$

In general, $W_{\kappa, \mu}$ is the acceptable physical solution which is finite at infinity. In the problem under consideration we must choose a linear combination of the two, due to the fact that both are finite in between the plates. The exact mode functions are

$$
\begin{aligned}
\phi_{\kappa}(u)= & u^{-\frac{1}{4}}\left[A\left(\omega, k_{\perp}\right) W_{\kappa, \frac{1}{4}}(u)\right. \\
& \left.+B\left(\omega, k_{\perp}\right) M_{\kappa, \frac{1}{4}}(u)\right] \mathrm{e}^{-i \omega t-i k_{x} x-i k_{y} y} .
\end{aligned}
$$

The asymptotic form of (23) for small value of $\lambda_{0}$ can be written as follows (see Appendix A):

$$
\begin{aligned}
\phi_{\kappa}(z)= & C_{0}\left(\omega, k_{\perp}\right)\left(g_{00} S(z)\right)^{-\frac{1}{4}} \sin \\
& \times\left(\int_{0}^{z} \sqrt{S} \mathrm{~d} z+\phi_{0}\right) \mathrm{e}^{-i \omega t-i k_{x} x-i k_{y} x},
\end{aligned}
$$

in which $S=\frac{\omega^{2}}{g_{00}}-k_{\perp}^{2}, g_{00}=1+2 \lambda_{0} z$. In the next section we use this asymptotic form to extract the mode frequencies for both Neumann and Dirichlet boundary conditions on the plates.

\section{Mode frequencies for Neumann and Dirichlet boundary conditions for the scalar field}

We put the approximation $\int \sqrt{S} \mathrm{~d} z \simeq \sqrt{b} z+\frac{a}{4 \sqrt{b}} z^{2}, a=$ $-2 \omega^{2} \lambda_{0}, b=\omega^{2}-k_{\perp}^{2}$ into (24). From the Dirichlet boundary condition $\phi_{\kappa}(z=0)=0$ we have $\phi_{0}=0$ and from $\phi_{\kappa}(z=$ $\left.l+\gamma_{1} l+\frac{1}{2} \lambda_{1} l^{2}\right)=0$ we have

$\int_{0}^{l+\gamma_{1} l+\frac{1}{2} \lambda_{1} l^{2}} \sqrt{S} \mathrm{~d} z=n \pi$.

After careful expansion of (25), the mode frequencies proved to satisfy the following relation:

$$
\begin{gathered}
\omega^{2}\left\{1-\lambda_{0}\left[l+\gamma_{1} l+\frac{1}{2} \lambda_{1} l^{2}\right]\right\} \\
=\sqrt{k_{\perp}^{2}+\left(\frac{n \pi}{l+\gamma_{1} l+\frac{1}{2} \lambda_{1} l^{2}}\right)^{2}} .
\end{gathered}
$$

Note that the factor $l+\gamma_{1} l+\frac{1}{2} \lambda_{1} l^{2}=l_{P}=\int_{0}^{l} \sqrt{g_{33}} \mathrm{~d} z=$ $\int_{0}^{l} \sqrt{1+2 \gamma_{1}+2 \lambda_{1} z} \mathrm{~d} z$ is nothing but the proper distance between the plates and thus we have

$\omega=\omega_{0}\left(1+\lambda_{0} \frac{l_{p}}{2}\right)$

in which $\omega_{0}=\sqrt{k_{\perp}^{2}+\left(\frac{n \pi}{l_{P}}\right)^{2}}, n=0,1,2, \ldots$ denotes proper (or the corresponding flat space) mode frequencies in the local Lorentz frame of an observer comoving with the plates. As stated in the previous section, the final mode frequencies will be obtained by multiplication of the factor $1+\gamma_{0}$ due to rescaling of time during the gauge transformation (9). So we have the following final mode frequencies inside the Casimir apparatus for the spacetime (7):

$\omega=\omega_{0}\left(1+\gamma_{0}+\lambda_{0} \frac{l_{p}}{2}\right)$

Mode frequencies are influenced only by $g_{00}$ component of the metric from the point of view of a proper observer.

The Neumann boundary condition $\left.\partial_{z} \phi\right|_{z=0}=0$ imposed on (24) gives $\phi_{0}=\frac{\pi}{2}$ :

$$
\begin{aligned}
\left.\frac{\mathrm{d} \phi}{\mathrm{d} z}\right|_{z=0} & =\left.0 \Rightarrow \tan \left(\int_{0}^{0} \sqrt{S} \mathrm{~d} z+\phi_{0}\right)\right|_{z=0} \\
& =\left.\frac{4 \sqrt{S}\left(g_{00} S\right)}{\frac{\mathrm{d}}{\mathrm{d} z}\left(g_{00} S\right)}\right|_{z=0}=\frac{4 b}{O\left(\lambda_{0}\right)} \rightarrow \infty
\end{aligned}
$$

Another Neuman boundary condition, $\left.\partial_{z} \phi\right|_{z=l}=0$, leads to

$$
\begin{aligned}
\left.\frac{\mathrm{d} \phi}{\mathrm{d} z}\right|_{z=l} & =\left.0 \Rightarrow \cot \left(\int_{0}^{l} \sqrt{S} \mathrm{~d} z\right)\right|_{z=l} \\
& =\left.\frac{4 \sqrt{S}\left(g_{00} S\right)}{\frac{\mathrm{d}}{\mathrm{d} z}\left(g_{00} S\right)}\right|_{z=l}=\frac{4 b}{O\left(\lambda_{0}\right)} \rightarrow \infty,
\end{aligned}
$$

which in turn results in (26) and (27) again. 


\section{Generalization of the formalism when the electromagnetic field is present inside the plates}

The electromagnetic field has two physical degrees of freedom and it is well known that in the Rindler spacetime the electromagnetic field can be represented in terms of two scalar fields satisfying the Klein-Gordon equation separately [31]. The photon propagator and the energy-momentum tensor of the electromagnetic field in a weak gravitational of the Fermi spacetime have also been obtained in [15-19]. However, in [15-19], the computations have been done through a lengthy and cumbersome method of the Green functions. In a paper by the author [24], it is shown that the energy density (i.e. the 00 component of the energy-momentum tensor) of the electromagnetic field in Fermi spacetime is exactly the same as the energy density of the two scalar fields mentioned in [24]. The method was used without any reference to the Green function method frequently used in the literature. Here, we briefly review the relationship between the two scalar fields and the electromagnetic field in Fermi spacetime.

The spin one vector field in curved spacetime in the Lorenz gauge satisfies

$\square A^{\mu}+R_{v}^{\mu} A^{v}=0, \quad \nabla_{\mu} A^{\mu}=0, \quad \mu=0,1,2,3$.

It can be shown that in the Fermi metric, the Ricci tensor satisfies $R_{\mu \nu}=O\left(\lambda^{2}\right)$ and the second term of the wave equation (31) must be ignored. Furthermore, because the metric (10) is spatially flat, the Lorenz gauge in (31) can be broken into two independent parts [31]:

$\nabla^{a} A_{a}=0, \quad a=0,3 \equiv(t, z)$,

$\nabla^{i} A_{i}=0, \quad i=1,2 \equiv(x, y)$.

Here

$A_{i}=\varepsilon_{i j} \nabla^{j} \phi, \quad A_{a}=\varepsilon_{a b} \nabla^{b} \psi$,

and

$\epsilon_{i j}=\left(\begin{array}{cc}0 & 1 \\ -1 & 0\end{array}\right), \quad \epsilon_{a b}=\left(\begin{array}{cc}0 & 1+\lambda z \\ -1-\lambda z & 0\end{array}\right)$.

We know also that both $\psi$ and $\phi$ satisfy the Klein-Gordon equation separately (see the appendix in [24]). The boundary condition for the electric field on the plates is $\mathbf{E}_{\perp}(z=0)=$ $E_{z}(z=0)=0$ and $\mathbf{E}_{\perp}(z=l)=E_{z}(z=l)=0$, which in turn can be recast into boundary conditions on $\psi$ and $\phi$ according to Eq. (33). In [24] it has been shown that boundary conditions for the electric and magnetic fields return a Dirichlet boundary condition on $\phi$ and a Neumann boundary condition on $\psi$. We have shown in the previous section that both of these conditions will lead to the same frequency shift. As a result the mode frequencies in (28) are also valid for the electromagnetic field.

\section{The energy-momentum tensor}

This section has three subsections. In the first subsection, the electromagnetic energy-momentum tensor will be represented in terms of the energy-momentum of the scalar fields mentioned in (33). In the other two sections the energymomentum tensor of the scalar and vector fields will be calculated.

6.1 The relationship between the energy-momentum tensor of the scalar and vector fields

The vacuum expectation value of the quantum energymomentum tensor is defined as

$\left\langle 0\left|T_{\mu \nu}\right| 0\right\rangle=\sum_{\mathbf{k}} T_{\mu \nu}\left[\phi_{\mathbf{k}}, \phi_{\mathbf{k}}^{*}\right]$.

The classical energy-momentum tensors for the scalar and vector fields are

$$
\begin{aligned}
T_{\mu \nu}^{\text {Scalar }}= & \partial_{\mu} \phi \partial_{\nu} \phi-\frac{1}{2} g_{\mu \nu} g^{\lambda \theta} \partial_{\lambda} \phi \partial_{\theta} \phi, \\
T_{\mu \nu}^{\text {vector }}= & T_{\mu \nu}^{\text {Ghost }}+T_{\mu \nu}^{\text {Gauge }}+T_{\mu \nu}^{\text {Maxwell }}, \\
T_{\mu \nu}^{\text {Ghost }}= & \zeta^{-1}\left[A_{\mu} A_{; \rho \nu}^{\rho}-A_{; \rho \mu}^{\rho} A_{\nu}\right. \\
& \left.-g_{\mu \nu}\left\{A^{\rho} A_{; \theta \rho}^{\theta}+\frac{1}{2}\left(A_{; \rho}^{\rho}\right)^{2}\right\}\right], \\
T_{\mu \nu}^{\text {Gauge }}= & -c_{; \mu}^{*} c_{; \nu}-c_{; \nu}^{*} c_{; \mu}-g_{\mu \nu} g^{\lambda \theta} c_{; \lambda} c_{; \theta \theta}, \\
T_{\mu \nu}^{\text {Maxwell }}= & \frac{1}{4} g_{\mu \nu} F^{\lambda \theta} F_{\lambda \theta}-F_{\mu \theta} F_{\theta \nu} .
\end{aligned}
$$

As is well known, in the quantum level, the contributions of ghost and gauge fields in the electromagnetic energymomentum tensor cancel each other [15-19] and we are only concerned with the Maxwell sector of the energy-momentum

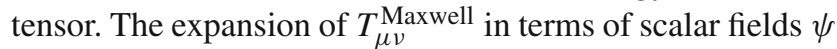
and $\phi$ shows that (see Appendix B)

$$
\begin{aligned}
T_{00}^{\text {Maxwell }}= & -\frac{1}{2}\left(E^{2}+\left(1+2 \lambda_{0} z\right) B^{2}\right), \\
T_{0 i}^{\text {Maxwell }}= & -(\vec{E} \times \vec{B})_{i}, \\
T_{i j}^{\text {Maxwell }}= & \frac{1}{2}\left(1-2 \lambda_{0} z\right)\left(E^{2}+\left(1+2 \lambda_{0} z\right) B^{2}\right) g_{i j} \\
& -\left(1-2 \lambda_{0} z\right) E_{i} E_{j}-B_{i} B_{j},
\end{aligned}
$$

in which $E^{2}=g_{i j} E^{i} E^{j}, B^{2}=g_{i j} B^{i} B^{j}, g_{i j}=-\delta_{i j}, E_{i}=$ $F_{0 i}, F_{i j}=\varepsilon_{i j k} B^{k}$. Using $F_{\mu \nu}=A_{\mu, \nu}-A_{\nu, \mu}$ and (32) we have

$$
\begin{aligned}
& F_{\tau x}=-i k_{x} \sqrt{1+2 \lambda z} \partial_{z} \psi+\omega k_{y} \phi, \\
& F_{\tau y}=-i k_{y} \sqrt{1+2 \lambda z} \partial_{z} \psi-\omega k_{x} \phi, \\
& F_{\tau z}=-\sqrt{1+2 \lambda z} k_{\perp}^{2} \psi, \\
& F_{y z}=i k_{x} \partial_{z} \phi+\omega(1-2 \lambda z) \sqrt{1+2 \lambda z} k_{y} \psi, \\
& F_{x z}=-i k_{y} \partial_{z} \phi+\omega(1+2 \lambda z) \sqrt{1+2 \lambda z} k_{x} \psi, \\
& F_{x y}=k_{\perp}^{2} \phi, \\
& \vec{E}=\left(F_{\tau x}, F_{\tau y}, F_{\tau z}\right), \\
& \vec{B}=\left(-F_{y z}, F_{x z},-F_{x y}\right),
\end{aligned}
$$


in which we have used the general form of the wave function (12). Quadratic products of fields $E^{2}, B^{2}, E_{i} E_{j}$, and $B_{i} B_{j}$ produce terms like $\psi \partial_{z} \phi^{*}, \phi \partial_{z} \psi^{*}, \phi \psi^{*}$, which have no contribution when the expectation value is taken, because of the fact that $\psi$ and $\phi$ are not correlated and belong to independent Hilbert spaces. We calculate the $0-0$ component of the energy-momentum tensor for the electromagnetic field:

$$
\begin{aligned}
\left\langle 0\left|T_{00}^{\phi}\right| 0\right\rangle= & \sum_{\omega} \int \mathrm{d}^{2} k_{\perp}\left\{\frac{1}{2}\left(\omega^{2}+(1+2 \lambda z) k_{\perp}^{2}\right)|\phi|^{2}\right. \\
+ & \left.\frac{1}{2}(1+2 \lambda z)\left|\partial_{z} \phi\right|^{2}\right\}, \\
\left\langle 0\left|T_{00}^{\mathrm{Max}}\right| 0\right\rangle= & -\sum_{\omega} \int \mathrm{d}^{2} k_{\perp}\left\{\frac { 1 } { 2 } \left(\left\langle 0\left|E^{2}\right| 0\right\rangle\right.\right. \\
& \left.\left.+(1+2 \lambda z)\left\langle 0\left|B^{2}\right| 0\right\rangle\right)\right\} \\
= & \sum_{\omega} \int \mathrm{d}^{2} k_{\perp}\left\{\frac { 1 } { 2 } \left(\left\langle 0\left|F_{\tau x}^{2}+F_{\tau y}^{2}+F_{\tau z}^{2}\right| 0\right\rangle\right.\right. \\
& \left.\left.+(1+2 \lambda z)\left\langle 0\left|F_{y z}^{2}+F_{x z}^{2}+F_{x y}^{2}\right| 0\right\rangle\right)\right\} \\
= & \sum_{\omega} \int \mathrm{d}^{2} k_{\perp}\left\{k _ { \perp } ^ { 2 } \left[\frac { 1 } { 2 } \left\{\left(\omega^{2}+(1+2 \lambda z) k_{\perp}^{2}\right)|\phi|^{2}\right.\right.\right. \\
& \left.+(1+2 \lambda z)\left|\partial_{z} \phi\right|^{2}\right\} \\
& +\frac{1}{2}\left\{\left(\omega^{2}+(1+2 \lambda z) k_{\perp}^{2}\right)|\psi|^{2}\right. \\
& \left.\left.\left.+(1+2 \lambda z)\left|\partial_{z} \psi\right|^{2}\right\}\right]\right\} \\
= & k_{\perp}^{2}\left[\left\langle 0\left|T_{00}^{\phi}\right| 0\right\rangle+\left\langle 0\left|T_{00}^{\psi}\right| 0\right\rangle\right] .
\end{aligned}
$$

After a lengthy but straightforward calculation, the other components of the energy-momentum tensor in both sides are related to each other as follows:

$$
\begin{aligned}
\left\langle 0\left|T_{00}^{\text {Maxwell }}\right| 0\right\rangle & =k_{\perp}^{2}\left\{\left\langle 0\left|T_{00}^{\phi}\right| 0\right\rangle+\left\langle 0\left|T_{00}^{\psi}\right| 0\right\rangle\right\}, \\
\left\langle 0\left|T_{11}^{\text {Maxwell }}\right| 0\right\rangle & =-k_{\perp}^{2}\left\{\left\langle 0\left|T_{11}^{\phi}\right| 0\right\rangle+\left\langle 0\left|T_{11}^{\psi}\right| 0\right\rangle\right\} \\
& +2(1-2 \lambda z) k_{x}^{2}\left\{\left\langle 0\left|T_{00}^{\phi}\right| 0\right\rangle+\left\langle 0\left|T_{00}^{\psi}\right| 0\right\rangle\right\}, \\
\left\langle 0\left|T_{22}^{\text {Maxwell }}\right| 0\right\rangle & =-k_{\perp}^{2}\left\{\left\langle 0\left|T_{22}^{\phi}\right| 0\right\rangle+\left\langle 0\left|T_{22}^{\psi}\right| 0\right\rangle\right\} \\
& +2(1-2 \lambda z) k_{y}^{2}\left\{\left\langle 0\left|T_{00}^{\phi}\right| 0\right\rangle+\left\langle 0\left|T_{00}^{\psi}\right| 0\right\rangle\right\}, \\
\left\langle 0\left|T_{33}^{\text {Maxwell }}\right| 0\right\rangle & =k_{\perp}^{2}\left\{\left\langle 0\left|T_{33}^{\phi}\right| 0\right\rangle+\left\langle 0\left|T_{33}^{\psi}\right| 0\right\rangle\right\}, \\
\left\langle 0\left|T_{01}^{\text {Maxwell }}\right| 0\right\rangle & =k_{\perp}^{2}\left\{\left\langle 0\left|T_{01}^{\phi}\right| 0\right\rangle+\left\langle 0\left|T_{01}^{\psi}\right| 0\right\rangle\right\}, \\
\left\langle 0\left|T_{02}^{\text {Maxwell }}\right| 0\right\rangle & =k_{\perp}^{2}\left\{\left\langle 0\left|T_{02}^{\phi}\right| 0\right\rangle+\left\langle 0\left|T_{02}^{\psi}\right| 0\right\rangle\right\}, \\
\left\langle 0\left|T_{03}^{\text {Maxwell }}\right| 0\right\rangle & =-k_{\perp}^{2}\left\{\left\langle 0\left|T_{03}^{\phi}\right| 0\right\rangle+\left\langle 0\left|T_{03}^{\psi}\right| 0\right\rangle\right\}, \\
\left\langle 0\left|T_{12}^{\text {Maxwell }}\right| 0\right\rangle & =-k_{\perp}^{2}\left\{\left\langle 0\left|T_{12}^{\phi}\right| 0\right\rangle+\left\langle 0\left|T_{12}^{\psi}\right| 0\right\rangle\right\} \\
& +2(1-2 \lambda z)\left\{\left\langle 0\left|T_{00}^{\phi}\right| 0\right\rangle+\left\langle 0\left|T_{00}^{\psi}\right| 0\right\rangle\right\}, \\
\left\langle 0\left|T_{23}^{\text {Maxwell }}\right| 0\right\rangle & =-k_{\perp}^{2}\left\{\left\langle 0\left|T_{23}^{\phi}\right| 0\right\rangle+\left\langle 0\left|T_{23}^{\psi}\right| 0\right\rangle\right\}, \\
\left\langle 0\left|T_{13}^{\text {Maxwell }}\right| 0\right\rangle & =-k_{\perp}^{2}\left\{\left\langle 0\left|T_{13}^{\phi}\right| 0\right\rangle+\left\langle 0\left|T_{13}^{\psi}\right| 0\right\rangle\right\} .
\end{aligned}
$$

Note that $k_{\perp}^{2}$ can be absorbed into $C$.

6.2 Energy density for Dirichlet and Neumann scalar fields

This section is devoted to the calculation of the energy density for the Casimir apparatus via the direct method without any reference to the traditional Green function method. We use the approximations

$$
\begin{aligned}
& \left(g_{00} S\right)^{-\frac{1}{4}} \simeq 1-\left(\frac{\lambda}{2}+\frac{a}{4 b}\right) z, \\
& \int \sqrt{S} \mathrm{~d} z \simeq \sqrt{b} z+\frac{a}{4 \sqrt{b}} z^{2}, \\
& \quad a=-2 \omega^{2} \lambda, \quad b=\omega^{2}-k_{\perp}^{2}, \\
& \sin \left(\sqrt{b} z+\frac{a}{4 \sqrt{b}} z^{2}\right)=\sin (\sqrt{b} z)+\frac{a}{4 \sqrt{b}} z^{2} \cos (\sqrt{b} z), \\
& \cos \left(\sqrt{b} z+\frac{a}{4 \sqrt{b}} z^{2}\right)=\cos (\sqrt{b} z)-\frac{a}{4 \sqrt{b}} z^{2} \sin (\sqrt{b} z) .
\end{aligned}
$$

We expand the wave function (24) and find up to first order in $\lambda$

$$
\begin{aligned}
Z(z)= & Z_{0}\left\{\left[1-\left(\frac{\lambda}{2}+\frac{a}{4 b}\right) z\right] \sin (\sqrt{b} z)\right. \\
& \left.+\frac{a}{4 \sqrt{b}} z^{2} \cos (\sqrt{b} z)\right\}, \text { Dirichlet, } \\
Z(z)= & Z_{0}\left\{\left[1-\left(\frac{\lambda}{2}+\frac{a}{4 b}\right) z\right] \cos (\sqrt{b} z)\right. \\
& \left.-\frac{a}{4 \sqrt{b}} z^{2} \sin (\sqrt{b} z)\right\}, \text { Newmann }
\end{aligned}
$$

$Z_{0}$ can be absorbed also in $C_{0}$.

The energy density is defined by $\varepsilon=n^{\mu} n^{\nu}\left\langle 0\left|T_{\mu \nu}^{\phi}\right| 0\right\rangle$ where $n^{\mu}$ is the lapse vector normal to the hypersurface $z=$ constant, i.e. $n^{\mu}=\partial_{z} z=\frac{1}{1+2 \lambda_{0} z}(0,0,0,1)$. The mean energy density thus has the following form:

$$
\begin{aligned}
\bar{\varepsilon}= & \frac{1}{l} \int_{0}^{l} n^{\mu} n^{\nu}\left\langle 0\left|T_{\mu \nu}^{\phi}\right| 0\right\rangle \mathrm{d} z \\
= & \sum_{\omega} \int \mathrm{d}^{2} k_{\perp} \frac{1}{l} \int_{0}^{l}\left\{\frac{1}{2}\left(\omega^{2}+\left(1+2 \lambda_{0} z\right) k_{\perp}^{2}\right) Z(z)^{2}\right. \\
& \left.+\frac{1}{2}\left(1+2 \lambda_{0} z\right)\left|\partial_{z} Z(z)\right|^{2}\right\} \frac{\mathrm{d} z}{\left(1+2 \lambda_{0} z\right)} \\
\equiv & \sum_{\omega} \int \mathrm{d}^{2} k_{\perp} H\left(\omega, k_{\perp}\right) .
\end{aligned}
$$

Calculating the factor $H\left(\omega, k_{\perp}\right)$ for both wave functions in (45) results in

$$
\begin{aligned}
& H^{\text {Dirichlet }}\left(\omega, k_{\perp}\right)=C^{2}\left\{\frac{1}{2} \omega^{2} F_{1}+\frac{1}{2} k_{\perp}^{2} F_{2}+\frac{1}{2} F_{3}\right\}, \\
& F_{1}=\left\{\frac{z}{2}-\frac{\sin (2 \sqrt{b} z)}{4 \sqrt{b}}-\left(3 \lambda+\frac{a}{2 b}\right)\left[\frac{z^{2}}{4}-\frac{z \sin (2 \sqrt{b} z)}{4 \sqrt{b}}-\frac{\cos (2 \sqrt{b} z)}{8 b}\right]\right. \\
& \left.\left.+\frac{a}{4 \sqrt{b}}\left[\frac{z}{2 b} \sin (2 \sqrt{b} z)+\left(\frac{1}{2 b}-z^{2}\right) \frac{\cos (2 \sqrt{b} z)}{2 \sqrt{b}}\right]\right\}\right\}_{\alpha}^{\beta}, \\
& F_{2}=\left\{\frac{z}{2}-\frac{\sin (2 \sqrt{b} z)}{4 \sqrt{b}}-\left(\lambda+\frac{a}{2 b}\right)\left[\frac{z^{2}}{4}-\frac{z \sin (2 \sqrt{b} z z}{4 \sqrt{b}}-\frac{\cos (2 \sqrt{b} z)}{8 b}\right]\right. \\
& \left.\left.+\frac{a}{4 \sqrt{b}}\left[\frac{z}{2 b} \sin (2 \sqrt{b} z)+\left(\frac{1}{2 b}-z^{2}\right) \frac{\cos (2 \sqrt{b} z)}{2 \sqrt{b}}\right]\right\}\right\}_{\alpha}^{\beta}, \\
& F_{3}=\left\{b\left[\frac{z}{2}+\frac{\sin (2 \sqrt{b} z)}{4 \sqrt{b}}+\left(\frac{a}{2 b}-\lambda\right)\left[\frac{z^{2}}{4}+\frac{z \sin (2 \sqrt{b} z)}{4 \sqrt{b}}+\frac{\cos (2 \sqrt{b} z)}{8 b}\right]\right]\right. \\
& \left.\quad-\frac{a \sqrt{b}}{4}\left[\frac{z}{2 b} \sin (2 \sqrt{b} z)+\left(\frac{1}{2 b}-z^{2}\right) \frac{\cos (2 \sqrt{b} z z)}{2 \sqrt{b}}\right]+\left(\frac{\lambda}{4}+\frac{a}{8 b}\right) \cos (2 \sqrt{b} z)\right\}\left.\right|_{\alpha} ^{\beta},
\end{aligned}
$$


$H^{\text {Neuman }}\left(\omega, k_{\perp}\right)=C^{2}\left\{\frac{1}{2} \omega^{2} F_{1}+\frac{1}{2} k_{\perp}^{2} F_{2}+\frac{1}{2} F_{3}\right\}$,

$F_{1}=\left\{\frac{z}{2}+\frac{\sin (2 \sqrt{b} z)}{4 \sqrt{b}}-\left(3 \lambda+\frac{a}{2 b}\right)\left[\frac{z^{2}}{4}+\frac{z \sin (2 \sqrt{b} z)}{4 \sqrt{b}}+\frac{\cos (2 \sqrt{b} z)}{8 b}\right]\right.$

$\left.-\frac{a}{4 \sqrt{b}}\left[\frac{z}{2 b} \sin (2 \sqrt{b} z)+\left(\frac{1}{2 b}-z^{2}\right) \frac{\cos (2 \sqrt{b} z)}{2 \sqrt{b}}\right]\right\}\left.\right|_{\alpha} ^{\beta}$,

$F_{2}=\left\{\frac{z}{2}+\frac{\sin (2 \sqrt{b} z)}{4 \sqrt{b}}-\left(\lambda+\frac{a}{2 b}\right)\left[\frac{z^{2}}{4}+\frac{z \sin (2 \sqrt{b} z)}{4 \sqrt{b}}+\frac{\cos (2 \sqrt{b} z)}{8 b}\right]\right.$

$\left.-\frac{a}{4 \sqrt{b}}\left[\frac{z}{2 b} \sin (2 \sqrt{b} z)+\left(\frac{1}{2 b}-z^{2}\right) \frac{\cos (2 \sqrt{b} z)}{2 \sqrt{b}}\right]\right\}\left.\right|_{\alpha} ^{\beta}$,

$F_{3}=\left\{b\left[\frac{z}{2}-\frac{\sin (2 \sqrt{b} z)}{4 \sqrt{b}}+\left(\frac{a}{2 b}-\lambda\right)\left[\frac{z^{2}}{4}-\frac{z \sin (2 \sqrt{b} z)}{4 \sqrt{b}}-\frac{\cos (2 \sqrt{b} z)}{8 b}\right]\right]\right.$

$\left.+\frac{a \sqrt{b}}{4}\left[\frac{z}{2 b} \sin (2 \sqrt{b} z)+\left(\frac{1}{2 b}-z^{2}\right) \frac{\cos (2 \sqrt{b} z)}{2 \sqrt{b}}\right]-\left(\frac{\lambda}{4}+\frac{a}{8 b}\right) \cos (2 \sqrt{b} z)\right\}\left.\right|_{\alpha} ^{\beta}$.

Simplification of the terms like $\cos (2 \sqrt{b} z)$ and $\sin (2 \sqrt{b} z)$ is possible using the fact that in the boundaries the wave function (24) must vanish and

$$
\int_{0}^{z} \sqrt{S} \mathrm{~d} z=n \pi-\Phi_{0} \Rightarrow \sqrt{b} z+\frac{a}{4 \sqrt{b}} z^{2}=n \pi-\Phi_{0},
$$

in which $z$ takes one of two boundary values $z=0$ and $z=l+\gamma_{1} l+\frac{1}{2} \lambda_{1} l^{2}$. Equation (47) results in

$\sin (2 \sqrt{b} z)=-\frac{a}{2 \sqrt{b}} z^{2}-\Phi_{0}, \quad \cos (2 \sqrt{b} z) \simeq 1$,

and the final result is as follows:

$H^{\text {Neuman }}\left(\omega, k_{\perp}\right)=C^{2} \frac{\omega^{2}}{2}\left\{l-\left[\lambda+\frac{a}{4 b}\right]\left(\beta^{2}-\alpha^{2}\right)\right\}$.

Exactly the same $H$ is obtained for the Dirichlet boundary condition. The constant $C$ was defined in (7) and can be determined simply,

$$
\begin{aligned}
C^{2}= & \frac{1}{2(2 \pi)^{2} \omega}\left\{\int _ { 0 } ^ { l } \left\{\left[1-\left(2 \lambda+\frac{a}{2 b}\right) z\right] \sin ^{2}(\sqrt{b} z)\right.\right. \\
& \left.\left.+\frac{a}{4 \sqrt{b}} z^{2} \sin (2 \sqrt{b} z)\right\} \mathrm{d} z\right\}^{-1}, \text { Dirichlet, } \\
C^{2}= & \frac{1}{2(2 \pi)^{2} \omega}\left\{\int _ { 0 } ^ { l } \left\{\left[1-\left(2 \lambda+\frac{a}{2 b}\right) z\right] \cos ^{2}(\sqrt{b} z)\right.\right. \\
& \left.\left.-\frac{a}{4 \sqrt{b}} z^{2} \sin (2 \sqrt{b} z)\right\} \mathrm{d} z\right\}^{-1}, \text { Neumann. }
\end{aligned}
$$

Just like $H$, the constant $C$ has the same form for both boundary conditions up to first order in $\lambda$,

$$
C^{2}=\frac{1}{2(2 \pi)^{2} \omega}\left\{\frac{l}{2}-\left(2 \lambda+\frac{a}{2 b}\right) \frac{\left(\beta^{2}-\alpha^{2}\right)}{4}\right\}^{-1} .
$$

The final result for the energy density finally is

$$
\begin{aligned}
\bar{\varepsilon} & =\sum_{\omega} \int \mathrm{d}^{2} k_{\perp} \frac{\omega}{2(2 \pi)^{2}} \\
& =\sum_{\omega} \int \mathrm{d}^{2} k_{\perp} \frac{\omega_{0}\left(1+\gamma_{0}+\lambda_{0} \frac{l_{P}}{2}\right)}{2(2 \pi)^{2}}
\end{aligned}
$$

$$
=\left(1+\gamma_{0}+\lambda_{0} \frac{l_{P}}{2}\right) \sum_{\omega} \int \mathrm{d}^{2} k_{\perp} \frac{\omega_{0}}{2(2 \pi)^{2}},
$$

in which $\bar{\varepsilon}_{0}=\sum_{\omega} \int \mathrm{d}^{2} k_{\perp} \frac{\omega_{0}}{2(2 \pi)^{2}}$ is the corresponding flat spacetime Casimir energy density, $\bar{\varepsilon}_{0}=-\frac{\pi^{2}}{1440 l^{4}}$ [32].

Up to now, we have shown that for Neumann and Dirichlet boundary conditions we have the relation $\left\langle 0\left|T_{00, \text { Fermi }}^{\psi}\right| 0\right\rangle=$ $\left(1+\gamma_{0}+\lambda_{0} \frac{l_{P}}{2}\right)\left\langle 0\left|T_{00, \text { Flat }}^{\psi}\right| 0\right\rangle$ between flat and curved energy density contents of the Casimir apparatus in the weak spacetime of the metric (7).

\subsection{Energy density for the electromagnetic field}

Using the first equation of (41) it is evident that the same shift in the energy density as obtained in (52) holds for the electromagnetic field:

$$
\begin{aligned}
\left\langle 0\left|T_{00}^{\text {Maxwell }}\right| 0\right\rangle= & \left\langle 0\left|T_{00}^{\phi}\right| 0\right\rangle+\left\langle 0\left|T_{00}^{\psi}\right| 0\right\rangle \\
= & \left\{\left\langle 0\left|T_{00, \text { flat }}^{\phi}\right| 0\right\rangle+\left\langle 0\left|T_{00, \text { flat }}^{\psi}\right| 0\right\rangle\right\}(1 \\
& \left.+\gamma_{0}+\lambda_{0} \frac{l_{P}}{2}\right) \\
= & \left\{-\frac{\pi^{2}}{1440 l^{4}}-\frac{\pi^{2}}{1440 l^{4}}\right\}\left(1+\gamma_{0}+\lambda_{0} \frac{l_{P}}{2}\right) \\
= & -\frac{\pi^{2}}{720 l^{4}}\left(1+\gamma_{0}+\lambda_{0} \frac{l_{P}}{2}\right) .
\end{aligned}
$$

In the next section we will analyze some well-known weak gravitational fields and find the parameters $\gamma_{0}, \gamma_{1}, \lambda_{0}, \lambda_{1}$ in each case.

\subsection{Notes on the divergences}

The problem of the divergences near a perfect generic conductor first studied systematically by Deutsch and Candelas [33]. They found that the energy-momentum tensor near the surface behaves like

$\left\langle 0\left|T_{00}\right| 0\right\rangle=\frac{c_{1}}{\epsilon^{4}}+\frac{c_{2}}{\epsilon^{3}}+\cdots$,

where $c_{1}, c_{2}$ are constants and $\epsilon$ is the distance from the surface of the ideal boundary. In the case where there is a conformal invariance in the action, $c_{1}=0$. The divergence originates from the unphysical nature of the classical ideal conductor boundary conditions. It has been shown [33] that we can remove the infinities of the total energy (and not the energy-momentum tensor) of the plates using the zeta function regularization unless we have the case that the zeta function has poles itself. On the other hand, the cutoff regularization method suggests the removal of the divergences ad hoc although in this method there still remains a logarithmic ambiguity $[34,35]$ in the energy density. For the imperfect conductors (more realistic boundary conditions) we can easily remove the divergences introducing some suitable cutoff frequencies although the boundary effect may become quit large (but they remain finite) $[33,36]$. 
Now the question is: what happens when we go to curved spacetime? Are the surface divergences of the energymomentum tensor ignorable in the semi-classical Einstein's equations? The answer is negative according to [33]. In $[34,35]$, however, the authors found a way to get rid of the surface divergences of the Einstein field equations for the case the boundary is a parallelepiped. They have used a suitable cutoff along with the so-called Estrada-Kanwal distribution theory of asymptotics to regularize/renormalize the infinities and show that the energy-momentum tensor near a plane boundary, as a source, converges to a consistent theory when the cutoff is removed. Remarkably, the process of curing the divergences in curved spacetime has been continued by Milton et al. [36] where they have shown that most of the energy between the plates is restored near the surfaces and a part of it resides exactly on the plates. They finally have shown that these energies respond to gravity just like any other finite energy following the newtonian relation $F=m a=-M g$. This is expected as these large energies are simply a part of the total energy of the system.

We show here that, in our case, the divergences are not present in the first order of approximation that we have used here and they appear only in the higher orders of approximation. To do so, we write down the explicit structure of possible divergences in the Einstein's field equations along the lines depicted in [32]. The one-loop effective action $W$ for the semi-classical theory of gravity is

$R_{\mu \nu}-\frac{1}{2} R g_{\mu \nu}+\Lambda g_{\mu \nu}=-\frac{8 \pi G}{c^{4}}\left\langle\left|T_{\mu \nu}\right|\right\rangle$,

$W=\int \sqrt{-g} L_{\text {eff. }}(x) \mathrm{d}^{n} x$,

$L_{\text {eff. }}(x)=\frac{i}{2} \lim _{x \rightarrow x^{\prime}} \int_{m^{2}}^{\infty} \mathrm{d} m^{2} G_{F}^{\text {D.S. }}\left(x, x^{\prime}\right)$,

in which $G_{F}^{\text {D.S. }}\left(x, x^{\prime}\right)$ is the DeWitt-Schwinger-Feynmann's propagator. Using the DeWitt-Schwinger representation of the action, the asymptotic expansion of $L_{\text {eff. }}$ is as follows:

$L_{\mathrm{eff} .}(x)=\frac{1}{2}(4 \pi)^{-\frac{n}{2}}\left(\frac{m}{\mu}\right)^{n-4} \sum_{j=0}^{\infty} a_{j}(x) m^{4-2 j} \Gamma\left(j-\frac{n}{2}\right)$,

in which $\mu$ is a length scale to fix the dimensional issues. The potentially divergent part of the effective action (the first three terms) [32] reads

$$
\begin{aligned}
L_{\mathrm{div}}= & -(4 \pi)^{\frac{n}{2}}\left\{\frac{1}{n-4}+\frac{1}{2}\left[\gamma+\ln \frac{m^{2}}{\mu^{2}}\right]\right\} \\
& \times\left[\frac{1}{n(n-2)} 4 m^{2} a_{0}-\frac{1}{(n-2)} 2 m^{2} a_{1}+a_{2}\right] .
\end{aligned}
$$

Here $m$ is the mass of the scalar field ( $m=0$ in our case). The coefficients $a_{0}, a_{1}, a_{2}$ are

$$
\begin{aligned}
a_{0}(x)= & 1, a_{1}(x)=\frac{1}{6} R, a_{2}(x)=\frac{1}{180} R_{\alpha \beta \gamma \delta} R^{\alpha \beta \gamma \delta} \\
& -\frac{1}{180} R_{\alpha \beta} R^{\alpha \beta}-\frac{1}{6} \square R+\frac{1}{72} R^{2},
\end{aligned}
$$

in which $R_{\alpha \beta \gamma \delta}$ is the Riemann curvature tensor. The total gravitational lagrangian density can be shown to have the form (see (6.49) in [32])

$$
\begin{aligned}
\mathscr{L}= & -\left(A+\frac{\Lambda_{B}}{8 \pi G_{B}}\right)+\left(B+\frac{1}{16 \pi G_{B}}\right) R \\
& -\frac{1}{(4 \pi)^{n / 2}}\left\{\frac{1}{n-4}+\frac{1}{2}\left[\gamma+\ln \frac{m^{2}}{\mu^{2}}\right]\right\} a_{2}, \\
A= & \frac{4 m^{4}}{(4 \pi)^{\frac{n}{2}} n(n-2)}\left\{\frac{1}{n-4}+\frac{1}{2}\left[\gamma+\ln \frac{m^{2}}{\mu^{2}}\right]\right\}, \\
B= & \frac{m^{2}}{(4 \pi)^{3 \frac{n}{2}}(n-2)}\left\{\frac{1}{n-4}+\frac{1}{2}\left[\gamma+\ln \frac{m^{2}}{\mu^{2}}\right]\right\} .
\end{aligned}
$$

However, in the massless case, the only non-vanishing potentially ultraviolent term is the one related to $a_{2}$ in (52) and $A, B$ in (52) vanish (see (6.101) in [32]). Our calculation for the metric (7) shows that $a_{2}$ is of second order of approximation:

$$
\begin{aligned}
R_{1212}= & R_{1313}=-\frac{\lambda_{0} \lambda_{1}}{4\left(1+2 \gamma_{1}+2 \lambda_{1} z\right)}=O\left(\lambda_{0}^{2}\right), \\
R_{1414}= & \frac{\lambda_{0}^{2}}{4\left(1+2 \gamma_{0}+2 \lambda_{0} z\right)}+\frac{\lambda_{0} \lambda_{1}}{4\left(1+2 \gamma_{1}+2 \lambda_{1} z\right)}=O\left(\lambda_{0}^{2}\right), \\
& -2 R_{2323}=R_{2424}=R_{3434}=-\frac{\lambda_{1}^{2}}{2\left(1+2 \gamma_{1}+2 \lambda_{1} z\right)} \\
= & O\left(\lambda_{1}^{2}\right), \\
R_{11}= & R_{22}=R_{33} \simeq O\left(\lambda_{0}^{2}\right), R_{44} \simeq O\left(\lambda_{0}^{2}\right), \\
R \quad \simeq & O\left(\lambda_{0}^{2}\right), \quad \square R \simeq O\left(\lambda_{0}^{2}\right) .
\end{aligned}
$$

So the total lagrangian density (59) reduces to the standard bare density $\frac{1}{16 \pi G_{B}} R$. In conclusion, the potentially divergent term $a_{2}$ vanishes within the first order of approximation.

\section{Examples: finding coefficients $\gamma_{0}, \gamma_{1}, \lambda_{0}, \lambda_{1}$}

In this section a number of spacetimes are investigated and the parameters appearing in (28), (52), and (53) will be found. To this end, we will try to find their weak field form according to (3), (4), and (7).

7.1 Electromagnetic Casimir energy density for the far field limit of the Kerr spacetime

Recently Bezerra et al. [8] studied the apparatus for the scalar fields in the weak field limit of the Kerr spacetime in the equatorial plane. In that work the apparatus co-rotates with the local angular velocity of the spacetime, i.e. the measurements had been assumed to be done in the point of view of a zero angular momentum observer (ZAMO). They found the metric inside the apparatus through some two stage successive approximation method as follows: 


$$
\begin{aligned}
\mathrm{d} s^{2} \approx & \left(1+2 b \Phi_{0}\right) \mathrm{d} t^{2}-\left(1-2 \Phi_{0}\right)\left[\mathrm{d} x^{2}+\mathrm{d} y^{2}\right. \\
& \left.+\mathrm{d} z^{2}\right] \text { 0th order of approximation, } \\
\mathrm{d} s^{2} \approx & (1+2 b \lambda z) \mathrm{d} t^{2}-(1-2 \lambda z)\left[\mathrm{d} x^{2}+\mathrm{d} y^{2}\right. \\
& \left.+\mathrm{d} z^{2}\right] \text { first order of approximation. }
\end{aligned}
$$

Here $b=1-2 a \Omega_{0}, \Phi_{0}=-\frac{G M}{R}$, and $\lambda=-\frac{G M}{R^{2}} . a$ is the angular momentum per mass and $\Omega_{0}$ the local angular velocity of the Kerr spacetime. The authors set $\Phi_{0}=0$ for the first order of approximations. This does not work because $\lambda$ is related to $\Phi_{0}$ through $\gamma=-R \Phi_{0}$. Putting one of them equal to zero forces the other one to vanish also. Evidently, in the first order of approximations we must keep both $\Phi_{0}$ and $\gamma$ and the metric must be written as follows instead of (61):

$\mathrm{d} s^{2} \approx \mathrm{d} t^{2}-\left[\mathrm{d} x^{2}+\mathrm{d} y^{2}+\mathrm{d} z^{2}\right]$ 0th order of approximation,

$\mathrm{d} s^{2} \approx\left[1+2 b\left(\Phi_{0}+\gamma z\right)\right] \mathrm{d} t^{2}-\left[1-2\left(\Phi_{0}+\gamma z\right)\right]\left[\mathrm{d} x^{2}\right.$

$\left.+\mathrm{d} y^{2}+\mathrm{d} z^{2}\right]$ first order of approximation.

Taking the above comment and the metric (62) into account, we find $\gamma_{0}=b \Phi_{0}, \lambda_{0}=b \gamma=-b R \Phi_{0}, \gamma_{1}=\Phi_{0}, \lambda_{1}=$ $\gamma=-b R \Phi_{0}$, and we arrive at the following relations for the electromagnetic Casimir energy density and the mode frequencies:

$\omega=\omega_{0}\left[1+b \Phi_{0}+b \frac{\gamma l_{P}}{2}\right]$,

$\left\langle 0\left|T_{00}^{\mathrm{Maxwell}}\right| 0\right\rangle=-\frac{\pi^{2}}{720 l^{4}}\left[1+b \Phi_{0}+b \frac{\gamma l_{P}}{2}\right]$.

The scalar field Casimir energy density inside the apparatus which has been sketched in Eq. (32) in [8] must also be corrected as follows:

$\omega=\omega_{0}\left[1+b \Phi_{0}+b \frac{\gamma l_{P}}{2}\right]$,

$\left\langle 0\left|T_{00}^{\text {scalar }}\right| 0\right\rangle=-\frac{\pi^{2}}{1440 l^{4}}\left[1+b \Phi_{0}+b \frac{\gamma l_{P}}{2}\right]$.

The far field limit of the Schwarzschild spacetime is also covered by setting $b=1$

\subsection{The Fermi spacetime}

The Fermi spacetime is described by the following metric:

$\mathrm{d} s^{2}=(1+2 a z) \mathrm{d} t^{2}-\mathrm{d} x^{2}-\mathrm{d} y^{2}-\mathrm{d} z^{2}$.

The importance of this metric is that it is traditionally recognized as the spacetime of a static accelerating observer near the surface of the source of a constant gravitational field [29]. Comparison of this metric to the general metric in (7) gives $\gamma_{0}=0, \lambda_{0}=a, \gamma_{1}=\lambda_{1}=0$ :

$\omega=\omega_{0}\left[1+\frac{a l_{P}}{2}\right]$,

$\left\langle 0\left|T_{00}^{\text {Maxwell }}\right| 0\right\rangle=-\frac{\pi^{2}}{720 l^{4}}\left[1+\frac{a l_{P}}{2}\right]$,

$\left\langle 0\left|T_{00}^{\text {scalar }}\right| 0\right\rangle=-\frac{\pi^{2}}{1440 l^{4}}\left[1+\frac{a l_{P}}{2}\right]$.
The above energy densities are exactly the results (5.2) in [15-19], (3.4) in [22], and (5.4) in [21].

7.3 The Hořava-Lifshitz gravity with a cosmological constant

The Hořava-Lifshitz (HL) gravity [37] is a renormalizable theory of gravity that is invariant under the Lifshitz scaling transformation $\mathbf{x} \rightarrow b \mathbf{x}, t \rightarrow b^{z} t$. These transformations manifestly break the space and time covariance. The anisotropy between space and time, in turn, may affect the Casimir effect as well. It is interesting to investigate the vacuum characteristics of the theory. Recently, the effect of the HL theory on the Casimir energy of the apparatus has been studied in [38]. The authors recommended to set a constraint on spacetime anisotropies in such a way that the Casimir energy modifications remain within the experimental bounds. Recently in $[10,11]$ the same problem considered in a curved spacetime in the context of a spherical symmetric solution of the HL theory. The finite temperature Casimir energy in spacetime (7) has been analyzed by the author in [39]. The following weak field limit for the HL theory has been calculated:

$$
\begin{aligned}
\mathrm{d} s^{2}= & \left\{1+\left[2 \frac{\widehat{M}}{R}+\frac{3 \widehat{M}^{2}}{2 R^{2}}-\frac{\widehat{M}^{2}}{2 \widehat{\omega} R^{4}}\right]\right. \\
& \left.+\left[-2 \frac{\widehat{M}}{R^{2}}-\frac{3 \widehat{M}^{2}}{2 R^{3}}+\frac{\widehat{M}^{2}}{2 \widehat{\omega} R^{5}}\right] z\right\} \mathrm{d} t^{2} \\
& -\left\{1+\left[-\frac{\widehat{M}}{R}+\frac{\widehat{M}^{2}}{4 R^{2}}+\frac{\widehat{M}^{2}}{4 \widehat{\omega} R^{4}}\right]\right. \\
& \left.+\left[\frac{\widehat{M}}{R^{2}}-\frac{\widehat{M}^{2}}{4 R^{3}}-\frac{\widehat{M}^{2}}{4 \widehat{\omega} R^{5}}\right] z\right\}\left(\mathrm{d} x^{2}+\mathrm{d} y^{2}+\mathrm{d} z^{2}\right)
\end{aligned}
$$

where $\widehat{M}=M\left(1+\frac{\Lambda}{\omega}\right)$. This spacetime is the weak field limit of Park's spherical symmetric solution to the IR limit of the HL theory in the presence of a cosmological constant $[11,40] . \Lambda$ may play the same role as the cosmological constant but it is not necessarily a small parameter and $\omega$ is a constant frequently used to regulate the UV limit of the HL theory. Comparison between (67) and the general metric (7) shows

$$
\begin{aligned}
& \gamma_{0} \approx \frac{M\left(1+\frac{\Lambda}{\omega}\right)}{R}, \lambda_{0} \approx-\frac{M\left(1+\frac{\Lambda}{\omega}\right)}{R^{2}}, \gamma_{1} \approx \frac{M\left(1+\frac{\Lambda}{\omega}\right)}{2 R} \\
& \lambda_{1} \approx-\frac{M\left(1+\frac{\Lambda}{\omega}\right)}{2 R^{2}} .
\end{aligned}
$$

Another spacetime, which is a solution to the HL theory without cosmological constant is the Kehagias-Sfetsos (KS) solution. This spacetime has been discussed in [10] to obtain the energy density of the apparatus and is as follows:

$$
\mathrm{d} s^{2}=f_{\mathrm{KS}} \mathrm{d} t^{2}-f_{\mathrm{KS}}^{-1} \mathrm{~d} \rho^{2}-\rho^{2} \mathrm{~d} \Omega^{2}
$$


where $\left.f_{\mathrm{KS}}=1+\omega \rho^{2}\left(1-\sqrt{(} 1+\frac{4 M}{\omega \rho^{3}}\right)\right)$. $\rho$ is a radial coordinate and $\omega$ is the free parameter of the HL theory. Putting $\Lambda=0$ in the Park solution one recovers the KS solution and so the coefficients in Eq. (68) are also valid for the KS solution.

\section{Conclusion}

We analyzed the energy density of a Casimir apparatus consisting of two nearby conducting parallel plates in a general weak gravitational field. The metric in Eq. (7) denotes the deviation of the weak gravitational field from flat spacetime inside the apparatus. We transformed the metric (7) through a gauge transformation into the Fermi metric and then solved the Klein-Gordon equation exactly. The mode frequencies were found for the scalar field inside the apparatus for both Neumann and Dirichlet boundary conditions in terms of the weak gravitational field parameters $\gamma_{0}, \gamma_{1}, \lambda_{0}, \lambda_{1}$. This result was shown to be valid also for the electromagnetic field in Sect. 5. The energy density of the apparatus was found for both scalar and electromagnetic fields in terms of the weak field parameters. Some examples of weak gravitational fields were analyzed in Sect. 7. Specially the electromagnetic energy density and mode frequencies in the far field limit of the Kerr spacetime in its equatorial plane were obtained. The weak field limit of the Hořava-Lifshitz gravity with a cosmological constant was also investigated and the weak field parameters were sketched. Consistency of the results with the literature was checked by considering the Fermi metric.

Acknowledgments The author would like to thank University of Tehran for supporting this project under the grants provided by the research council.

Open Access This article is distributed under the terms of the Creative Commons Attribution 4.0 International License (http://creativecomm ons.org/licenses/by/4.0/), which permits unrestricted use, distribution, and reproduction in any medium, provided you give appropriate credit to the original author(s) and the source, provide a link to the Creative Commons license, and indicate if changes were made.

Funded by SCOAP ${ }^{3}$.

\section{Appendix A: Asymptotic form of the wave function}

In this section we find an explicit and simple asymptotic form for the wave function. As is apparent we need to have an asymptotic expansion with both argument and first parameter large. The Whittaker functions have such an expansion in terms of the Airy functions [41]:

$$
\begin{aligned}
& W_{\kappa, \mu}(4 \kappa x)=2^{4 / 3} \sqrt{\pi} \kappa^{\kappa+1 / 6}\left(\frac{x \zeta}{x-1}\right)^{\frac{1}{4}} \\
& \quad \times\left\{A i\left[(4 \kappa)^{\frac{2}{3}} \zeta\right] \sum_{n=0}^{\infty} \frac{A_{n}(\zeta)}{(4 \kappa)^{2 n}}+\frac{A i^{\prime}\left[(4 \kappa)^{\frac{2}{3}} \zeta\right]}{(4 \kappa)^{\frac{4}{3}}} \sum_{n=0}^{\infty} \frac{B_{n}(\zeta)}{(4 \kappa)^{2 n}}\right\}
\end{aligned}
$$

where $\zeta$ is defined as

$\frac{4}{3}(-\zeta)^{\frac{3}{2}}=\cos ^{-1}(\sqrt{x})-\sqrt{x-x^{2}}$,

and in our case $4 \kappa x=\frac{k_{\perp}}{\lambda} g_{00}$ and so

$x=\frac{k_{\perp}^{2}}{\omega^{2}} g_{00}=\frac{k_{\perp}^{2}}{\omega^{2}}(1+2 \lambda z), \quad x<1 \quad$ or

$S \equiv \frac{\omega^{2}}{g_{00}}-k_{\perp}^{2}>0$.

If $\kappa \rightarrow \infty$ then both the argument and the first parameter go to infinity. As $\kappa \propto \lambda^{-1}$ the second term in the bracket is of $\operatorname{order} \lambda^{\frac{4}{3}}$ and must be ignored to stay within the first order of expansion in $\lambda$. Also the summation in the first term reduces to only the $n=0$ term and $A_{0}(\zeta)=$ constant. We have

$W_{\kappa, \mu}(4 \kappa x)=2^{4 / 3} \sqrt{\pi} \kappa^{\kappa+1 / 6}\left(\frac{x \zeta}{x-1}\right)^{\frac{1}{4}} A i\left[(4 \kappa)^{\frac{2}{3}} \zeta\right]$.

The argument of the above Airy function can be written

$$
\begin{aligned}
(4 \kappa)^{\frac{2}{3}} \zeta & =-\left\{3 \kappa\left[\cos ^{-1}(\sqrt{x})-\sqrt{x-x^{2}}\right]\right\}^{\frac{2}{3}} \\
& \equiv-v(x), \quad v \rightarrow \infty .
\end{aligned}
$$

The Airy function with large argument is as follows (Sect. 9.7 in [30]):

$$
\begin{aligned}
A i(-v) \sim v^{-\frac{1}{4}} & \left\{\cos \left(\frac{2}{3} v^{\frac{3}{2}}-\frac{\pi}{4}\right) \sum_{n=0}^{\infty}(-1)^{n} \frac{a_{2 n}}{\left(\frac{2}{3} v^{\frac{3}{2}}\right)^{2 n}}\right. \\
& \left.+\sin \left(\frac{2}{3} v^{\frac{3}{2}}-\frac{\pi}{4}\right) \sum_{n=0}^{\infty}(-1)^{n} \frac{a_{2 n+1}}{\left(\frac{2}{3} v^{\frac{3}{2}}\right)^{2 n+1}}\right\} .
\end{aligned}
$$

As $v^{\frac{3}{2}}=O\left(\lambda^{-1}\right)$, the $\sin ()$-part and $n>0$ in the $\cos ()$-part must be ignored also. The observation that

$$
\begin{aligned}
\cos ^{-1}(\sqrt{x})-\sqrt{x-x^{2}} & =-\int \sqrt{\frac{1-x}{x}} \mathrm{~d} x \\
& =-\frac{1}{k_{\perp}} \int \sqrt{S} \mathrm{~d} x=-\frac{1}{2 \kappa} \int \sqrt{S} \mathrm{~d} z
\end{aligned}
$$

and

$$
\left(\frac{x \zeta}{x-1}\right)^{\frac{1}{4}} \propto v^{\frac{1}{4}} S^{-\frac{1}{4}}
$$

moves the situation forward significantly:

$$
W_{\kappa, \mu} \propto S^{-\frac{1}{4}} \cos \left(\int \sqrt{S} \mathrm{~d} z+\phi_{0}\right), \quad \phi_{0}=\phi_{0}(\kappa, \omega) .
$$


The same process can be applied to the other Whittaker function $M_{\kappa, \mu}$, except that, according to [42], instead of the argument $(4 \kappa)^{\frac{2}{3}} \zeta$ in (A5) we have $(4 \kappa)^{\frac{2}{3}} \exp \left( \pm \frac{2 \pi}{3}\right) \zeta$. This difference changes the above process as follows:

$$
\begin{aligned}
\zeta & \rightarrow \mathrm{e}^{ \pm \frac{2 \pi}{3}} \zeta, \quad v \rightarrow \mathrm{e}^{ \pm \frac{2 \pi}{3}} v, \quad \frac{2}{3} v^{\frac{3}{2}} \rightarrow \frac{2}{3} v^{\frac{3}{2}} \mathrm{e}^{ \pm \pi i} \\
& =-\frac{2}{3} v^{\frac{3}{2}}
\end{aligned}
$$

$M_{\kappa, \mu} \propto S^{-\frac{1}{4}} \cos \left(\int \sqrt{S} \mathrm{~d} z+\phi_{1}\right), \quad \phi_{1}=\phi_{1}(\kappa, \omega)$.

As a result the total wave function in (22) when $\lambda \rightarrow 0$ reads

$$
\begin{aligned}
\phi_{\kappa}(u)= & C_{0}\left(\omega, k_{\perp}\right)\left(g_{00} S(z)\right)^{-\frac{1}{4}} \sin \left(\int \sqrt{S} \mathrm{~d} z+\phi_{2}\right) \\
& \times \mathrm{e}^{-i \omega t-i k_{x} x-i k_{y} y}
\end{aligned}
$$

where $\phi_{2}=\phi_{2}\left(\omega, k_{\perp}, A, B, \phi_{0}, \phi_{1}\right)$ and $A, B$ came from (22) and the following relation has been used:

$$
\begin{aligned}
& A \cos \left(\int \sqrt{S} \mathrm{~d} z+\phi_{0}\right)+B \cos \left(\int \sqrt{S} \mathrm{~d} z+\phi_{1}\right) \\
& =C \sin \left(\int \sqrt{S} \mathrm{~d} z+\phi_{2}\right) .
\end{aligned}
$$

\section{Appendix B: Computation of the components of the energy-momentum tensor}

In this section we find $T^{i j}$. The other components will be found by similar techniques. We do the computations in the Fermi spacetime, i.e. $g_{00}=1+2 \lambda z, g_{0 i}=0, g_{i j}=-\delta_{i j}$. Greek indices run from 0 to 3 and Latin indices from 1 to 3 . From (36) we have

$T_{\text {Maxwell }}^{i j}=\frac{1}{4} g^{i j} F^{\lambda \theta} F_{\lambda \theta}-F^{i \theta} F_{\theta}^{j}$.

The first term has the following form:

$$
\begin{aligned}
& F^{\lambda \theta} F_{\lambda \theta}=2 F^{0 i} F_{o i}+F^{i j} F_{i j}, \\
& F^{0 i} F_{o i}=g^{00} E^{i} E_{i}, \\
& F^{i j} F_{i j}=g^{\mu i} g^{\nu j} F_{\mu \nu} \varepsilon_{i j k} B^{k}=\cdots=-2 B^{2} .
\end{aligned}
$$

Here we have used $\varepsilon_{i j k} \varepsilon_{i j l}=2 \delta_{k l}$. The second term in (B1) simplifies as follows:

$$
\begin{aligned}
F^{i \theta} F_{\theta}^{j} & =F^{i 0} F_{0}^{j}+F^{i m} F_{m}^{j}, \\
F^{i 0} & =g_{00} E^{i}, \quad F_{0}^{j}=-E^{j}, \\
F^{i m} & =g^{i l} g^{m k} F_{l k}=g^{i l} g^{m k} \varepsilon l k n B^{n}, \\
F^{j} & =g^{a j} F_{a m}=g^{a j} \varepsilon a m b B^{b}, \\
F^{i m} F_{m}^{j} & =-g^{i l} g^{a j} \varepsilon_{m a b} \varepsilon_{m l n} B^{b} B^{n} \\
& =-g^{i l} g^{a j}\left[\delta_{a l} \delta_{b n}-\delta_{a n} \delta_{b l}\right] B^{b} B^{n} \\
& =\cdots=-g^{i j} B^{2}+B^{i} B^{j} .
\end{aligned}
$$

Based on (B1)-(B3) we find

$T_{\text {Maxwell }}^{i j}=\frac{1}{2} g^{i j}\left(g^{00} E^{2}+B^{2}\right)-g^{00} E^{i} E^{j}-B^{i} B^{j}$.

After lowering the indices we have finally

$T_{i j}^{\text {Maxwell }}=\frac{1}{2} g_{i j}\left(g^{00} E^{2}+B^{2}\right)-g^{00} E_{i} E_{j}-B_{i} B_{j}$.

Now we find $T_{0 i}$. From (36) we have

$T_{\text {Maxwell }}^{00}=\frac{1}{4} g^{00} F^{\lambda \theta} F_{\lambda \theta}-F^{0 \theta} F_{\theta}^{0}$,

in which the first term already has been obtained in (B2). The second term has also been obtained in (B3), and after lowering the indices again we have

$T_{00}^{\text {Maxwell }}=-\frac{1}{2}\left(E^{2}+g_{00} B^{2}\right)$.

Now we find $T_{0 i}$. From (36) we have

$$
\begin{aligned}
T_{\text {Maxwell }}^{0 i} & =\frac{1}{4} g^{0 i} F^{\lambda \theta} F_{\lambda \theta}-F^{0 \theta} F_{\theta}^{i} \\
& =-F^{0 j} F_{j}^{i}=-g_{00} E^{j} g^{m i} F_{m j} \\
& =-g_{00} g^{m i} \varepsilon_{m j k} E^{j} B^{k}=-g_{00} g^{m i}(\mathbf{E} \times \mathbf{B})_{m},
\end{aligned}
$$

from which, after lowering the indices again, we find

$T_{0 i}^{\mathrm{Maxwell}}=-(\mathbf{E} \times \mathbf{B})_{i}$.

\section{References}

1. K.A. Milton et al., J. Phys. A 40, 10935-10943 (2007)

2. S.A. Fulling, K.A. Milton, P. Parashar, A. Romeo, K.V. Shajesh, J. Wagner, Phys. Rev. D 76, 025004 (2007)

3. S. Lamoreaux, Phys. Rev. Lett. 78, 5 (1996)

4. G. Bressi et al., Phys. Rev. Lett. 88, 041804 (2002)

5. A. Jaffe, Phys. Rev. D 72, 021301 (2005)

6. L.H. Ford, Phys. Rev. D 14, 3304 (1976)

7. B.S. DeWitt, Phys. Rep. 19, 295 (1975)

8. V.B. Bezerra, H.F. Mota, C.R. Muniz, Phys. Rev. D 89, 044015 (2014)

9. F. Sorge, Phys. Rev. D 90, 084050 (2014)

10. C.R. Muniz, V.B. Bezerra, M.S. Cunha, Phys. Rev. D 88, 104035 (2013)

11. C.R. Muniz, V.B. Bezerra, M.S. Cunha, Ann. Phys. 359, 55-63 (2015)

12. B. Geyer, G.L. Klimchitskaya, V.M. Mostepanenko, Phys. Rev. A. 67, $062102(2003)$

13. M. Bordag, G.L. Klimchitskaya, U. Mohideen, V.M. Mostepanenko, Advances in Casimir Effect (Oxford University Press, Oxford, 2009)

14. M. Bordag, Proceedings of the Fourth Workshop on Quantum Field Theory Under the Influence of External conditions: the Casimir Effect 50 Years Later (World Scientific, Singapore, 1998)

15. G. Bimonte et al., Phys. Rev. D 74, 085011 (2006)

16. G. Bimonte et al., Phys. Rev. D 78, 024010 (2008)

17. G. Bimonte et al., Phys. Rev. D 75, 049904 (2007) (erratumibid.)

18. G. Bimonte et al., Phys. Rev. D 75, 089901 (2007). (erratum ibid.)

19. G. Bimonte et al., Phys. Rev. D 77, 109903 (2008). (erratum ibid.) 
20. F. Sorge, Class. Quantum. Grav. 22, 5109-5119 (2005)

21. G. Esposito, G.M. Napolitano, L. Rosa, Phys. Rev. D 77, 105011 (2008)

22. G.M. Napolitano, G. Esposito, L. Rosa, Phys. Rev. D 78, 107701 (2008)

23. M. Nouri-zonoz, B. Nazari, Phys. Rev. D 82, 044047 (2010)

24. B. Nazari, M. Nouri-zonoz, Phys. Rev. D 85, 044060 (2012)

25. J.S. Dowker, R. Critchley, J. Phys. A Math. Gen. 9, 535 (1976)

26. D. Lynden-Bell, M. Nouri-Zonoz, Rev. Mod. Phys. 70, 427 (1998)

27. L.D. Landao, E.M. Lifshitz, The Classical Theory of Fields (Pergamon Press, Oxford, 1987) (reprint)

28. W. Rindler, Relativity: Special. General and Cosmological (Cambridge University Press, Cambridge, 2006). (chapter 9)

29. C.W. Misner, K.S. Thorne, J.H. Wheeler, Gravitation (W.H. Freeman and Company, New York, 1973)

30. A.B. Olde Daalhuis, NIST Handbook of Mathematical Functions (Cambridge University Press, New York, 2010) (Ch. 13)

31. P. Deutsch, D. Candelas, Proc. R. Soc. Lond. A 354, 79-99 (1977)

32. N.D. Birrell, P.C.W. Davies, Quantum Field Theory in Curved Spacetime (Cambridge University Press, Cambridge, 1982)
33. P. Deutsch, D. Candelas, Phys. Rev. D 20, 3063 (1979) (79-99, 1977)

34. R. Estrada, S.A. Fulling, L. Kaplan, K. Kirsten, Z. Liu, K.A. Milton, J. Phys. A Math. Theor. 41, 164055 (2008)

35. R. Estrada, S.A. Fulling, F.D. Mera, J. Phys. A Math. Theor. 45, $455402(2012)$

36. K.A. Milton, K.V. Shajesh, S.A. Fulling, P. Parashar, Phys. Rev. D 89, 064027 (2014)

37. P. Hořava, Phys. Rev. D 79, 084008 (2009)

38. A.F. Ferrari et al., Mod. Phys. Lett. A 29, 1350052 (2013)

39. B. Nazari, Thermal corrections to the Casimir effect in a weak gravitational field (under review)

40. M. Park, JHEP 0909, 123 (2009)

41. F.W.J. Olver, Asymptotics and Special Functions (Academic Press, New York, 1974) (chapter 11, Ex. 7.3)

42. A. Erdlyi, C.A. Swanson, Asymptotic forms of Whittaker's confluent hypergeometric functions. Mem. Am. Math. Soc. 1957(25), 1-49 (1957) (equation 10.3) 